\title{
GLOBAL SYMMETRIES OF TIME-DEPENDENT SCHRÖDINGER EQUATIONS
}

\author{
Susumu Okubo \\ Department of Physics and Astronomy \\ University of Rochester \\ Rochester, NY 14627, USA
}

\begin{abstract}
Some symmetries of time-dependent Schrödinger equations for inverse quadratic, linear, and quadratic potentials have been systematically examined by using a method suitable to the problem. Especially, the symmetry group for the case of the linear potential turns out to be a semi-direct product $S L(2, R)$ (S) $T_{2}(R)$ of the $S L(2, R)$ with a two-dimensional real translation group $T_{2}(R)$. Here, the time variable $t$ transforms as $t \rightarrow t^{\prime}=(c t+d) /(a t+b)$ for real constants $a, b, c$, and $d$ satisfying $b c-a d=1$ with an accompanying transformation for the space coordinate $x$.
\end{abstract}

\section{Formulation}

Many solutions of Schrödinger equations are known ${ }^{1-4}$ ) (and earlier references quoted therein) to possess dynamical (or hidden) symmetries which are not apparent at first glance.

In a different approach, Eastwood ${ }^{5)}$ in his study of symmetry of Laplace equation has observed the following: Suppose that a pair of functions $U(\mathbf{x}, \boldsymbol{\partial})$ and $W(\mathbf{x}, \boldsymbol{\partial})$ of the coordinate $\mathbf{x}$ and its derivative $\boldsymbol{\partial}$ satisfies

$$
W(\mathbf{x}, \boldsymbol{\partial}) \Delta=\Delta U(\mathbf{x}, \boldsymbol{\partial}), \quad\left(\Delta=\sum_{j=1}^{n} \frac{\partial^{2}}{\partial x_{j}^{2}}\right)
$$

for the Laplacian $\Delta$. Then, if $\psi(\mathbf{x})$ is a solution of the Laplace equation $\Delta \psi(\mathbf{x})=0$, then so will be

$$
\psi^{\prime}(\mathbf{x})=U(\mathbf{x}, \boldsymbol{\partial}) \psi(\mathbf{x})
$$

The purpose of this note is to utilize an analogous method to systematically find global symmetries of time-independent Schrödinger equations. We consider the equation of motion of the form:

$$
\frac{\partial}{\partial t} \psi(t, \mathbf{x})=k\{\Delta-V(\mathbf{x})\} \psi(t, \mathbf{x})
$$

If $k$ is purely imaginary with $k=-i \hbar / 2 m$, then it describes the standard Schrödinger equation, while the case of $k$ being real implies a diffusion-type equation. In what 
follows, we will always assume that the parameter $k$ is either real or purely imaginary with $t$ and $\mathbf{x}$ being real unless it is stated otherwise.

Consider now a vector space of all suitably smooth functions of $t$ and $\mathbf{x}$, which may be complex. Suppose that we can find a pair of linear operators $U$ and $W$ in this space to satisfy the condition

$$
W\left\{\frac{\partial}{\partial t}-k \Delta+k V(\mathbf{x})\right\}=\left\{\frac{\partial}{\partial t}-k \Delta+k V(\mathbf{x})\right\} U .
$$

Then, if $\psi(t, \mathbf{x})$ satisfies Eq.(1.1), the new function given by

$$
\psi^{\prime}(t, \mathbf{x})=(U \psi)(t, \mathbf{x}) \equiv U \psi(t, \mathbf{x})
$$

will also obey the same relation, i.e. we have

$$
\frac{\partial}{\partial t} \psi^{\prime}(t, \mathbf{x})=k\{\Delta-V(\mathbf{x})\} \psi^{\prime}(t, \mathbf{x})
$$

Some explicit forms of $U$ and $W$ can be found as follows. Let us consider the coordinate transformation of form for $\mathbf{x}=\left(x_{1}, x_{2}, \ldots, x_{n}\right)$,

$$
\begin{aligned}
t \rightarrow t^{\prime} & =\phi(t) \quad, \\
x_{j} \rightarrow x_{j}^{\prime} & =F_{j}(t, \mathbf{x}) \quad, \quad(j=1,2, \ldots, n)
\end{aligned}
$$

for some differentiable functions $\phi(t)$ and $F_{j}(t, \mathbf{x})$ to be determined. The action of the linear operator $U$ to a function $\psi(t, \mathbf{x})$ is then assumed to be given as a multiplication of a function after the coordinate transformation, i.e.,

$$
\psi^{\prime}(t, \mathbf{x})=U \psi(t, \mathbf{x})=K(t, \mathbf{x}) \psi\left(t^{\prime}, \mathbf{x}^{\prime}\right)
$$

where $K(t, \mathbf{x})$ is a function of $t$ and $\mathbf{x}$ to be determined. When we note

$$
\begin{aligned}
\frac{\partial}{\partial t} & =\dot{\phi}(t) \frac{\partial}{\partial t^{\prime}}+\sum_{j=1}^{n} \frac{\partial F_{j}(t, \mathbf{x})}{\partial t} \frac{\partial}{\partial x_{j}^{\prime}} \\
\frac{\partial}{\partial x_{j}} & =\sum_{k=1}^{n} \frac{\partial F_{k}(t, \mathbf{x})}{\partial x_{j}} \frac{\partial}{\partial x_{k}^{\prime}}
\end{aligned}
$$

then we calculate

$$
\begin{aligned}
& \left\{\frac{\partial}{\partial t}-k \Delta+k V(\mathbf{x})\right\} \psi^{\prime}(t, \mathbf{x})=\left\{\frac{\partial}{\partial t}-k \Delta+k V(\mathbf{x})\right\}\left[K(t, \mathbf{x}) \psi\left(t^{\prime}, \mathbf{x}^{\prime}\right)\right] \\
& =\left\{\left[\frac{\partial}{\partial t}-k \Delta+k V(\mathbf{x})\right] K(t, \mathbf{x})\right\} \psi\left(t^{\prime}, \mathbf{x}^{\prime}\right)+
\end{aligned}
$$




$$
\begin{aligned}
& +K(t, \mathbf{x}) \sum_{j=1}^{n}\left\{\frac{\partial F_{j}(t, \mathbf{x})}{\partial t}-k \Delta F_{j}(t, \mathbf{x})\right. \\
& \left.-2 k \sum_{\ell=1}^{n} \frac{\partial \log K(t, \mathbf{x})}{\partial x_{\ell}} \frac{\partial F_{j}(t, \mathbf{x})}{\partial x_{\ell}}\right\} \frac{\partial}{\partial x_{j}^{\prime}} \psi\left(t^{\prime}, \mathbf{x}^{\prime}\right) \\
& +K(t, \mathbf{x})\left\{\dot{\phi}(t) \frac{\partial}{\partial t^{\prime}}-k \sum_{j, \ell=1}^{n}\left(\sum_{i=1}^{n} \frac{\partial F_{j}}{\partial x_{i}} \frac{\partial F_{\ell}}{\partial x_{i}}\right) \frac{\partial^{2}}{\partial x_{j}^{\prime} \partial x_{\ell}^{\prime}}\right\} \psi\left(t^{\prime}, \mathbf{x}^{\prime}\right) .
\end{aligned}
$$

If $F_{j}(t, \mathbf{x})$, and $K(t, \mathbf{x})$ satisfy relations:

$$
\begin{aligned}
& \text { (i) } \sum_{i=1}^{n} \frac{\partial F_{j}(t, \mathbf{x})}{\partial x_{i}} \frac{\partial F_{\ell}(t, \mathbf{x})}{\partial x_{i}}=\delta_{j \ell} \dot{\phi}(t)\left(\equiv \delta_{j \ell} \frac{d}{d t} \phi(t)\right), \\
& \text { (ii) }\left(\frac{\partial}{\partial t}-k \Delta\right) F_{j}(t, \mathbf{x})=2 k \sum_{\ell=1}^{n} \frac{\partial \log K(t, \mathbf{x})}{\partial x_{\ell}} \frac{\partial F_{j}(t, \mathbf{x})}{\partial x_{\ell}}, \\
& \text { (iii) }\left\{\frac{\partial}{\partial t}-k \Delta+k V(\mathbf{x})\right\} K(t, \mathbf{x})=k \dot{\phi}(t) V\left(\mathbf{x}^{\prime}\right) K(t, \mathbf{x}),
\end{aligned}
$$

then Eq. (1.7) becomes

$$
\left\{\frac{\partial}{\partial t}-k \Delta+k V(\mathbf{x})\right\} \psi^{\prime}(t, \mathbf{x})=\dot{\phi}(t) K(t, \mathbf{x})\left\{\frac{\partial}{\partial t^{\prime}}-k \Delta^{\prime}+k V\left(\mathbf{x}^{\prime}\right)\right\} \psi\left(t^{\prime}, \mathbf{x}^{\prime}\right)
$$

which reproduces Eq. (1.2) with actions of $U$ and $W$ given by

$$
\begin{aligned}
U \psi(t, \mathbf{x}) & =K(t, \mathbf{x}) \psi\left(t^{\prime}, \mathbf{x}^{\prime}\right) \\
W \hat{\psi}(t, \mathbf{x}) & =\dot{\phi}(t) K(t, \mathbf{x}) \hat{\psi}\left(t^{\prime}, \mathbf{x}^{\prime}\right)
\end{aligned}
$$

for any two functions $\psi$ and $\hat{\psi}$. For the present problem, we have

$$
\hat{\psi}(t, \mathbf{x})=\left(\frac{\partial}{\partial t}-k \Delta+k V(\mathbf{x})\right) \psi(t, \mathbf{x})
$$

Summarizing, we have proved the following Theorem.

\section{Theorem 1.1}

Let functions $\phi(t), F_{j}(t, \mathbf{x})$, and $K(t, \mathbf{x})$ satisfy Eqs. (1.8) with $t^{\prime}$ and $x_{j}^{\prime}$ being given by Eqs. (1.5). Then for any function $\psi(t, \mathbf{x})$ which satisfies Eq. (1.1), i.e.,

$$
\frac{\partial}{\partial t} \psi(t, \mathbf{x})=k\{\Delta-V(\mathbf{x})\} \psi(t, \mathbf{x})
$$


the new function given by

$$
\psi^{\prime}(t, \mathbf{x})=K(t, \mathbf{x}) \psi\left(t^{\prime}, \mathbf{x}^{\prime}\right)=K(t, \mathbf{x}) \psi\left(\phi(t), F_{j}(t, \mathbf{x})\right)
$$

is also a solution of the same generalized Schrödinger equation Eq. (1.1).

\section{Remark 1.2}

We may call the pair of linear operators, $(U, W)$ satisfying Eq. (1.2) be admissible. Then, for the second admissible pair $\left(U^{\prime}, W^{\prime}\right)$ their product $\left(U U^{\prime}, W W^{\prime}\right)$ is clearly also admissible. Moreover, the special pair $(1,1)$ acts as the identity. Therefore, a set of all admissible pairs form a semi-group. If the pair is invertible, then they present a symmetry group of Eq. (1.1). More explicitly, if the transformation with certain $V(\mathbf{x})$ are chosen as in Eq. (1.16), one gets a set $S$ of possible $\phi, F$, and $K$ with elements $s \in S$ parametrized by real numbers $a, b, \ldots$, i.e., $s=s(a, b, \ldots)$ and a set of solutions $\psi(t, \mathbf{x} ; a, b, \ldots)$. It will be shown in subsequent sections that the $s \in S$ and hence the corresponding solutions can be transformed into each other formally via e.g., $S L(2, R)$ depending upon $V(\mathbf{x})$.

We can modify Theorem 1.1 slightly as follows. Let $V_{0}(\mathbf{x})$ and $V(\mathbf{x})$ be two potentials, and suppose that the pair of linear operators $(U, W)$ now satisfies

$$
W\left\{\frac{\partial}{\partial t}-k \Delta+k V_{0}(\mathbf{x})\right\}=\left\{\frac{\partial}{\partial t}-k \Delta+k V(\mathbf{x})\right\} U
$$

instead Eq. (1.2). We can proceed exactly in the same way as in the previous case, and we prove the following theorem.

\section{Theorem 1.3}

Let functions $\phi(t), F_{j}(t, \mathbf{x})$ and $K(t, \mathbf{x})$ as in Theorem 1.1 except that Eq. (1.8c) is now replaced by

$$
\left\{\frac{\partial}{\partial t}-k \Delta+k V(\mathbf{x})\right\} K(t, \mathbf{x})=k \dot{\phi}(t) V_{0}\left(\mathbf{x}^{\prime}\right) K(t, \mathbf{x})
$$

Then, for any function $\psi_{0}(t, \mathbf{x})$ satisfying

$$
\left\{\frac{\partial}{\partial t}-k \Delta+k V_{0}(\mathbf{x})\right\} \psi_{0}(t, \mathbf{x})=0,
$$

the function given by

$$
\psi(t, \mathbf{x})=U \psi_{0}(t, \mathbf{x})=K(t, \mathbf{x}) \psi_{0}\left(t^{\prime}, \mathbf{x}^{\prime}\right)
$$

satisfies 


$$
\left\{\frac{\partial}{\partial t}-k \Delta+k V(\mathbf{x})\right\} \psi(t, \mathbf{x})=0
$$

\section{$\underline{\text { Remark } 1.4}$}

Examples satisfying Theorem 1.3 for $V_{0}(\mathbf{x})=0$ will be given in sections 3 and 4 . If $U^{-1}$ and $W^{-1}$ exist, then we can conversely express $\psi_{0}(t, \mathbf{x})$ in terms of $\psi(t, \mathbf{x})$.

Returning now to the original discussion of Theorem 1.1, it is in general difficult to find solutions of differential equations, Eqs. (1.8). However, for three cases of $V(\mathbf{x})$ being inverse square, linear, and quadratic potentials, we can solve them as follows. The explicit forms of $F_{j}(t, \mathbf{x})$ and $K(t, \mathbf{x})$ can then be assumed to be

$$
\begin{aligned}
t^{\prime} & =\phi(t) \\
x_{j}^{\prime} & =F_{j}(t, \mathbf{x})=\xi(t) x_{j}+f_{j}(t) \\
K(t, \mathbf{x}) & =\exp \left\{A(t)+\sum_{j=1}^{n} B_{j}(t) x_{j}+C(t) \mathbf{x}^{2}\right\},
\end{aligned}
$$

for some functions $\phi(t), \xi(t), f_{j}(t), A(t), B_{j}(t)$, and $C(t)$ of $t$ to be determined as in the following Proposition.

\section{Proposition 1.5}

Eqs. (1.8) will be satisfied for the ansätz Eqs. (1.16), if we have

$$
\begin{aligned}
\text { (i) } \dot{\phi}(t) & =\xi^{2}(t) \\
\text { (ii) } B_{j}(t) & =\frac{1}{2 k} \frac{\dot{f}_{j}(t)}{\xi(t)} \\
\text { (iii) } \quad C(t) & =\frac{1}{4 k} \frac{\dot{\xi}(t)}{\xi(t)},
\end{aligned}
$$

provided that $K(t, \mathbf{x})$ satisfies Eq. (1.8c), i.e.,

$$
\left\{\frac{\partial}{\partial t}-k \Delta+k V(\mathbf{x})\right\} K(t, \mathbf{x})=k \dot{\phi}(t) V\left(\mathbf{x}^{\prime}\right) K(t, \mathbf{x})
$$

We will solve these differential equations, Eqs. (1.17) and (1.18) in subsequent sections. The simplest case of the inverse quadratic potential will be discussed in section 2 , where the symmetry group is $S L(2, R)$. On the contrast, the symmetry of 
the linear potential is a larger one of $S L(2, R)$ (s) $T_{2}(R)$ which is the semi-direct product of $S L(2, R)$ with a real two-dimensional translation group $T_{2}(R)$. This will be presented in section 3. For the case of the quadratic potential in section 4 , the symmetry is now either a semi-group or a sub-group of $S L(2, C)$ (s) $T_{2}(C)$, depending upon choices of parameters involved in theory. In section 5, we will discuss the associated Lie algebras of these groups.

Last, we simply remark that the present method will also be applicable when the potential is time-dependent. In that case, we simply replace all $V(\mathbf{x}), V\left(\mathbf{x}^{\prime}\right)$, $V_{0}(\mathbf{x})$ and $V_{0}\left(\mathbf{x}^{\prime}\right)$ in Theorem 1.1 and 1.3 respectively by $V(t, \mathbf{x}), V\left(t^{\prime}, \mathbf{x}^{\prime}\right), V_{0}(t, \mathbf{x})$, and $V_{0}\left(t^{\prime}, \mathbf{x}^{\prime}\right)$. Also, we may generalize the present method by replacing $K(t, \mathbf{x})$ by $K\left(t, \mathbf{x}, \boldsymbol{\partial}, \partial_{t}\right)$ which may depend upon both space and time derivatives, $\boldsymbol{\partial}$ and $\partial_{t}\left(\equiv \frac{\partial}{\partial t}\right)$.

\section{Inverse Quadratic Potential}

In this section, we assume that the potential $V(\mathbf{x})$ is a homogenous function of $\mathbf{x}$ of degree -2 , i.e., it satisfies a identity

$$
V(\lambda \mathbf{x})=\frac{1}{\lambda^{2}} V(\mathbf{x})
$$

for any non-zero real number $\lambda$. For example, we may assume

$$
V(\mathbf{x})=\frac{\alpha}{x_{1}^{2}+x_{2}^{2}+\cdots+x_{n}^{2}}+\sum_{j=1}^{n} \frac{a_{j}}{\left(x_{j}\right)^{2}}+\sum_{j, k=1}^{n} \frac{b_{j k}}{\left(x_{j}-x_{k}\right)^{2}}
$$

etc. for some constants $\alpha, a_{j}$ and $b_{j k}$.

We must choose $f_{j}(t)=0$ with $x_{j}^{\prime}=\xi(t) x_{j}$ in this case. Then Eq. (2.1) implies the validity of

$$
V(\mathbf{x})=\xi^{2}(t) V\left(\mathbf{x}^{\prime}\right)=\dot{\phi}(t) V\left(\mathbf{x}^{\prime}\right)
$$

for $\lambda=\xi(t)$ so that Eq. (1.18) becomes

$$
\left\{\frac{\partial}{\partial t}-k \Delta\right\} K(t, \mathbf{x})=0
$$

which gives

$$
\begin{aligned}
& \dot{A}(t)=2 n k C(t), \\
& \dot{C}(t)=4 k(C(t))^{2} .
\end{aligned}
$$


Solving these with Eqs. (1.17) for $\xi(t)$ and $\phi(t)$, we find:

\section{Proposition 2.1}

For any function $\psi(t, \mathbf{x})$ satisfying

$$
\frac{\partial}{\partial t} \psi(t, \mathbf{x})=k\{\Delta-V(\mathbf{x})\} \psi(t, \mathbf{x})
$$

with the condition Eq. (2.1) for $V(\mathbf{x})$, a new function given by

$$
\psi^{\prime}(t, \mathbf{x})=\left(\frac{1}{a t+b}\right)^{\frac{n}{2}} \exp \left\{-\frac{a}{4 k(a t+b)} \mathbf{x}^{2}\right\} \psi\left(t^{\prime}, \mathbf{x}^{\prime}\right)
$$

also satisfies the same equation, i.e.,

$$
\frac{\partial}{\partial t} \psi^{\prime}(t, \mathbf{x})=k\{\Delta-V(\mathbf{x})\} \psi^{\prime}(t, \mathbf{x})
$$

where $t^{\prime}$ and $\mathrm{x}^{\prime}$ are given by

$$
t^{\prime}=\frac{c t+d}{a t+b} \quad, \quad \mathbf{x}^{\prime}=\frac{1}{a t+b} \mathbf{x} .
$$

Here, $a, b, c$, and $d$ are arbitrary real constants satisfying the condition

$$
b c-a d=1
$$

We will show next that the admissible pair $(U, W)$ of section 1 will offer infinite dimensional realizations of the $S L(2, R)$ group. For this purpose, it is more convenient to consider $2 \times 2 S L(2, R)$ matrix $M$ by

$$
M=\left(\begin{array}{ll}
c & d \\
a & b
\end{array}\right) \quad, \quad \operatorname{det} M=1 .
$$

Moreover, we collectively write the coordinates as

$$
Z=\{t, \mathbf{x}\}
$$

on which $M$ is assumed to act as

$$
M Z=M\{t, \mathbf{x}\}=\left\{t^{\prime}, \mathbf{x}^{\prime}\right\}=\left\{\frac{c t+d}{a t+b}, \frac{\mathbf{x}}{a t+b}\right\} .
$$

Since $K(t, \mathbf{x})$ depends upon parameters $a, b, c$, and $d$, we will write it as $K(t, \mathbf{x} \mid M)$ so that

$$
K(t, \mathbf{x} \mid M) \equiv K(Z \mid M)=\left(\frac{1}{a t+b}\right)^{\frac{n}{2}} \exp \left\{-\frac{a}{4 k(a t+b)} \mathbf{x}^{2}\right\}
$$


It is then easy to verify.

\section{Proposition 2.2}

For any two $S L(2, R)$ matrices $M$ and $M^{\prime}$, we have

$$
\begin{aligned}
& \text { (i) } \quad M\left(M^{\prime} Z\right)=\left(M M^{\prime}\right) Z \\
& \text { (ii) } K\left(Z \mid M^{\prime}\right) K\left(M^{\prime} Z \mid M\right)=K\left(Z \mid M M^{\prime}\right)
\end{aligned}
$$

where $M M^{\prime}$ implies the standard matrix product of $M$ and $M^{\prime}$.

The linear operators $U$ and $W$ introduced in section 1 also depend upon $M$. However, it is more convenient to rewrite them as $U\left(M^{-1}\right)$ and $W\left(M^{-1}\right)$ instead of $U(M)$ and $W(M)$ by the reason which will become clear shortly, so that Eq. (2.3) is rewritten as

$$
U\left(M^{-1}\right) \psi(Z)=K(Z \mid M) \psi(M Z)
$$

Note that

$$
M^{-1}=\left(\begin{array}{cc}
b & -d \\
-a & c
\end{array}\right) \quad \text { for } \quad M=\left(\begin{array}{cc}
c & d \\
a & b
\end{array}\right) .
$$

Then, Eqs. (2.11) are immediately translated into the following:

\section{Proposition 2.3}

Linear operators $U(M)$ and $W(M)$ satisfy

$$
\begin{aligned}
U\left(M M^{\prime}\right) & =U(M) U\left(M^{\prime}\right), \\
W\left(M M^{\prime}\right) & =W(M) W\left(M^{\prime}\right)
\end{aligned} .
$$

In other words, they offer (infinite dimensional) realizations of the $S L(2, R)$ group.

In ending this section, it may be of interest to note the following:

\section{Remark 2.4}

The transformation, Eqs. (2.5) contain both time translation and dilatation as special cases. If we choose

$$
M=\left(\begin{array}{ll}
1 & \lambda \\
0 & 1
\end{array}\right)
$$


then Eq. (2.5) gives the time translation,

$$
t \rightarrow t^{\prime}=t+\lambda \quad, \quad \mathbf{x} \rightarrow \mathbf{x}^{\prime}=\mathbf{x} .
$$

On the other side, the choice of

$$
M=\left(\begin{array}{cc}
c & 0 \\
0 & \frac{1}{c}
\end{array}\right) \quad, \quad(c \neq 0)
$$

leads to the dilatation

$$
t \rightarrow t^{\prime}=c^{2} t \quad, \quad \mathbf{x} \rightarrow \mathbf{x}^{\prime}=c \mathbf{x}
$$

\section{Remark 2.5}

For some subgroup of $S L(2, R)$, the linear operator $U(M)$ may possess a nontrivial fixed point in the function space. Consider one-dimensional case of $n=1$ with $V(\mathbf{x})=0$. The Jacobi's theta function $\theta_{1}(x \mid t)$ is given ${ }^{6)}$ by

$$
\theta_{1}(x \mid t)=i \sum_{n=-\infty}^{\infty}(-1)^{n} \exp \left\{i \pi\left(n-\frac{1}{2}\right)^{2} t+i \pi(2 n-1) x\right\}
$$

which satisfies one-dimensional Schrödinger equation

$$
4 \pi i \frac{\partial}{\partial t} \theta_{1}(x \mid t)=\frac{\partial^{2}}{\partial x^{2}} \theta_{1}(x \mid t)
$$

with $k=-i / 4 \pi$. Moreover, if $a, b, c, d$ are all integers with $b c-a d=1$, we then have the identity ${ }^{6)}$

$$
\theta_{1}\left(\frac{x}{a t+b} \mid \frac{c t+d}{a t+b}\right)=\epsilon(a t+b)^{\frac{1}{2}} \exp \left(\frac{i \pi a x^{2}}{a t+b}\right) \theta_{1}(x \mid t)
$$

where $\epsilon$ is a constant satisfying $\epsilon^{8}=1$, whose particular value depends upon the

specification of branches of $(a t+b)^{\frac{1}{2}}$ in the complex $t$-plane. Setting $\epsilon=1$. Eq. (2.17) is rewritten as

$$
U\left(M_{0}\right) \theta_{1}(x \mid t)=\theta_{1}(x \mid t)
$$

for any modular matrix $M_{0}$. Therefore, the $S L(2, R)$ orbit of $\theta_{1}(x \mid t)$ is the homogeneous space

$$
S L(2, R) / S L(2, Z)
$$

where $S L(2, Z)$ is the modular subgroup of $S L(2, R)$ in which all $a, b, c$, and $d$ are integers. 


\section{$\underline{\text { Remark 2.6 }}$}

For the one-dimensional case with $V(\mathbf{x})=0$, the symmetry group is actually a larger one of $S L(2, R)$ (S) $T_{2}(R)$ which is a semi-direct product of $S L(2, R)$ with a two-dimensional translation group $T_{2}(R)$. This will be shown as a special case of $\alpha=\beta=0$ in Eqs. (3.5)-(3.10) in the next section.

\section{Linear Potential}

In this section, we will consider the case of the linear potential. For a while, we restrict ourselves to one-dimensional space and set

$$
V(x)=\alpha+\beta x
$$

for constants $\alpha$ and $\beta$. Dropping all sub-indices such as $j$ 's in $f_{j}(t), B_{j}(t)$ etc. (since $n=1$ ), Eqs. (1.18) and (1.16c) then gives (with $\dot{A}(t) \equiv \frac{d}{d t} A(t)$ etc.)

$$
\begin{aligned}
& \text { (i) } \dot{C}(t)=4 k C^{2}(t) \\
& \text { (ii) } \dot{B}(t)=4 k B(t) C(t)+k \beta\left[\xi^{3}(t)-1\right], \\
& \text { (iii) } \dot{A}(t)=k\left\{B^{2}(t)+2 C(t)\right\}+k \alpha\left(\xi^{2}(t)-1\right)+k \beta \xi^{2}(t) f(t) .
\end{aligned}
$$

Together with Eqs. (1.17) which give $2 k B(t)=\dot{f}(t) / \xi(t)$ and $4 k C(t)=\dot{\xi}(t) / \xi(t)$, we can solve these equations. In this case, the general solution contains five independent real parameters. It is convenient for our purpose to parametrize them as

$$
\begin{aligned}
& \Lambda=\left\{M,\left(\begin{array}{l}
\mu \\
\nu
\end{array}\right)\right\} \\
& M=\left(\begin{array}{ll}
c & d \\
a & b
\end{array}\right), \quad \operatorname{det} M=1
\end{aligned}
$$

where $M$ is the real $2 \times 2$ unimodular matrix just as in the previous section, which acts now on 2-dimensional real vector $\left(\begin{array}{c}\mu \\ \nu\end{array}\right)$ in the parameter space. We write $C(t), B(t)$ etc. now as $C(t \mid \Lambda), B(t \mid \Lambda)$ etc. in order to indicate their dependence on parameters involved in $\Lambda$. We also rewrite $K(t, x)$ of section 1 as

$$
K(t, x \mid \Lambda)=\frac{1}{\sqrt{a t+b}} \exp \left\{A(t \mid \Lambda)+B(t \mid \Lambda) x+C(t \mid \Lambda) x^{2}\right\}
$$


where we changed however $A(t)$ there into $A(t \mid \Lambda)-\frac{1}{2} \log (a t+b)$ for simplicity. Then, their explicit forms are found to be

(i) $C(t \mid \Lambda)=-\frac{1}{4 k} \frac{a}{a t+b}$,

(ii) $B(t \mid \Lambda)=-\frac{\nu}{2 k} \frac{1}{a t+b}+\frac{k \beta}{2}\left\{\frac{2(c t+d)}{(a t+b)^{2}}-t-\frac{b t}{a t+b}\right\}$,

(iii) $\quad A(t \mid \Lambda)=-\frac{1}{4 k} \mu \nu+\alpha k\left(\frac{c t+d}{a t+b}-t\right)+\frac{\nu^{2}}{4 k} \frac{c t+d}{a t+b}$

$$
\begin{aligned}
& +k \beta\left\{\mu \frac{c t+d}{a t+b}-\nu\left[\left(\frac{c t+d}{a t+b}\right)^{2}-\frac{1}{2} \frac{t^{2}}{a t+b}\right]\right\} \\
& +k^{3} \beta^{2}\left\{\frac{2}{3}\left(\frac{c t+d}{a t+b}\right)^{3}+\frac{1}{12} t^{3}+\frac{b}{4} \frac{t^{3}}{a t+b}-\frac{t^{2}(c t+d)}{(a t+b)^{2}}\right\} .
\end{aligned}
$$

In Eq. (3.5c), the constant term $-\mu \nu / 4 k$ has been added to simplify the expression of $\omega\left(\Lambda, \Lambda^{\prime}\right)$ given in Eq. (3.16) shortly. We then have the following Proposition.

\section{Proposition 3.1}

For any function $\psi(t, x)$ satisfying

$$
\frac{\partial}{\partial t} \psi(t, x)=k\left\{\frac{\partial^{2}}{\partial x^{2}}-\alpha-\beta x\right\} \psi(t, x),
$$

the new wave function given by

$$
\psi^{\prime}(t, x)=K(t, x \mid \Lambda) \psi\left(t^{\prime}, x^{\prime}\right)
$$

satisfies the same, i.e.,

$$
\frac{\partial}{\partial t} \psi^{\prime}(t, x)=k\left\{\frac{\partial^{2}}{\partial x^{2}}-\alpha-\beta x\right\} \psi^{\prime}(t, x),
$$

where $t^{\prime}$ and $x^{\prime}$ are defined by

$$
\begin{aligned}
& t^{\prime}=\frac{c t+d}{a t+b} \\
& x^{\prime}=\frac{x}{a t+b}+\mu-\nu \frac{c t+d}{a t+b}+k^{2} \beta\left\{\left(\frac{c t+d}{a t+b}\right)^{2}-\frac{t^{2}}{a t+b}\right\}
\end{aligned}
$$


Next we will show that the underlying symmetry group is now the semi-direct product

$$
G=S L(2, R) \text { S } T_{2}(R)
$$

Let $\Lambda^{\prime} \in G$ with

$$
\Lambda^{\prime}=\left\{M^{\prime},\left(\begin{array}{l}
\mu^{\prime} \\
\nu^{\prime}
\end{array}\right)\right\} \quad, \quad \operatorname{det} M^{\prime}=1
$$

be the second generic element of $G$ in addition to $\Lambda$ given by Eqs. (3.3). We introduce the product $\Lambda \circ \Lambda^{\prime}$ by

$$
\Lambda \circ \Lambda^{\prime}=\left\{M M^{\prime},\left(\begin{array}{l}
\mu \\
\nu
\end{array}\right)+M\left(\begin{array}{l}
\mu^{\prime} \\
\nu^{\prime}
\end{array}\right)\right\}
$$

which can easily be verified to be associative and defines the desired group product of the group $G$. Note that the unit element 1 and the inverse $\Lambda^{-1}$ are then given by

$$
\begin{aligned}
1 & =\left\{\left(\begin{array}{ll}
1 & 0 \\
0 & 1
\end{array}\right),\left(\begin{array}{l}
0 \\
0
\end{array}\right)\right\}, \\
\Lambda^{-1} & =\left\{M^{-1},-M^{-1}\left(\begin{array}{l}
\mu \\
\nu
\end{array}\right)\right\},
\end{aligned}
$$

respectively. Again, it is convenient to write

$$
Z=\{t, x\}
$$

collectively for coordinates and assume the action of $\Lambda \in G$ to $Z$ to be given by

$$
\Lambda Z=\Lambda\{t, x\}=\left\{t^{\prime}, x^{\prime}\right\},
$$

in terms of $t^{\prime}$ and $x^{\prime}$ given by Eqs. (3.9). We then find

\section{Proposition 3.2}

We have

$$
\begin{aligned}
& \text { (i) } \Lambda\left\{\Lambda^{\prime} Z\right\}=\left(\Lambda \circ \Lambda^{\prime}\right) Z \\
& \text { (ii) } K\left(Z \mid \Lambda^{\prime}\right) K\left(\Lambda^{\prime} Z \mid \Lambda\right)=\exp \left\{\omega\left(\Lambda, \Lambda^{\prime}\right)\right\} K\left(Z \mid \Lambda \circ \Lambda^{\prime}\right) .
\end{aligned}
$$


Here, $\omega\left(\Lambda, \Lambda^{\prime}\right)$ is a constant given by

$$
\omega\left(\Lambda, \Lambda^{\prime}\right)=\frac{1}{4 k}\left\{(\mu a-\nu c) \mu^{\prime}+(\mu b-\nu d) \nu^{\prime}\right\}
$$

which satisfies the cycle condition

$$
\omega\left(\Lambda_{1}, \Lambda_{2}\right)+\omega\left(\Lambda_{1} \circ \Lambda_{2}, \Lambda_{3}\right)=\omega\left(\Lambda_{2}, \Lambda_{3}\right)+\omega\left(\Lambda_{1}, \Lambda_{2} \circ \Lambda_{3}\right)
$$

as well as

$$
\omega\left(\Lambda_{2}^{-1}, \Lambda_{1}^{-1}\right)=-\omega\left(\Lambda_{1}, \Lambda_{2}\right)
$$

for $\Lambda_{j} \in G(j=1,2,3)$.

The proof of this Proposition requires unfortunately long computations, although it is straightforward. First, we set

$$
\Lambda^{\prime} Z=\Lambda^{\prime}\{t, x\} \equiv\{\bar{t}, \bar{x}\}
$$

so that

$$
\begin{aligned}
& \bar{t}=\phi\left(t \mid \Lambda^{\prime}\right)=\frac{c^{\prime} t+d^{\prime}}{a^{\prime} t+b^{\prime}}, \\
& \bar{x}=\xi\left(t \mid \Lambda^{\prime}\right) x+f\left(t \mid \Lambda^{\prime}\right)
\end{aligned}
$$

with

$$
\begin{aligned}
& \xi\left(t \mid \Lambda^{\prime}\right)=\frac{1}{a^{\prime} t+b^{\prime}}, \\
& f\left(t \mid \Lambda^{\prime}\right)=\mu^{\prime}-\nu^{\prime} \frac{c^{\prime} t+d^{\prime}}{a^{\prime} t+b^{\prime}}+k^{2} \beta\left\{\left(\frac{c^{\prime} t+d^{\prime}}{a^{\prime} t+b^{\prime}}\right)^{2}-\frac{t^{2}}{a^{\prime} t+b^{\prime}}\right\} .
\end{aligned}
$$

Since

$$
K\left(Z \mid \Lambda^{\prime}\right)=\frac{1}{\sqrt{a^{\prime} t+b^{\prime}}} \exp \left\{A\left(t \mid \Lambda^{\prime}\right)+B\left(t \mid \Lambda^{\prime}\right) x+C\left(t \mid \Lambda^{\prime}\right) x^{2}\right\}
$$

and

$$
K\left(\Lambda^{\prime} Z \mid \Lambda\right)=\frac{1}{\sqrt{a \bar{t}+b}} \exp \left\{A(\bar{t} \mid \Lambda)+B(\bar{t} \mid \Lambda) \bar{x}+C(\bar{t} \mid \Lambda) \bar{x}^{2}\right\}
$$

we must now evaluate the product 


$$
K\left(Z \mid \Lambda^{\prime}\right) K\left(\Lambda^{\prime} Z \mid \Lambda\right)=\frac{1}{\sqrt{a^{\prime \prime} t+b^{\prime \prime}}} \exp \left\{A_{0}(t)+B_{0}(t) x+C_{0}(t) x^{2}\right\}
$$

with

$$
\begin{aligned}
& A_{0}(t)=A\left(t \mid \Lambda^{\prime}\right)+A(\bar{t} \mid \Lambda)+B(\bar{t} \mid \Lambda) f\left(t \mid \Lambda^{\prime}\right)+C(\bar{t} \mid \Lambda)\left[f\left(t \mid \Lambda^{\prime}\right)\right]^{2} \\
& B_{0}(t)=B\left(t \mid \Lambda^{\prime}\right)+B(\bar{t} \mid \Lambda) \xi\left(t \mid \Lambda^{\prime}\right)+2 C(\bar{t} \mid \Lambda) \xi\left(t \mid \Lambda^{\prime}\right) f\left(t \mid \Lambda^{\prime}\right) \\
& C_{0}(t)=C\left(t \mid \Lambda^{\prime}\right)+C(\bar{t} \mid \Lambda)\left[\xi\left(t \mid \Lambda^{\prime}\right)\right]^{2}
\end{aligned}
$$

Using expressions given in Eqs. (3.5), we can then verify the validity of Eq. (3.15b) after long calculations. For its computation, the following identities are however quite useful to simplify the proof. Let us set

$$
M^{\prime \prime}=M M^{\prime}=\left(\begin{array}{ll}
c & d \\
a & b
\end{array}\right)\left(\begin{array}{ll}
c^{\prime} & d^{\prime} \\
a^{\prime} & b^{\prime}
\end{array}\right)=\left(\begin{array}{ll}
c^{\prime \prime} & d^{\prime \prime} \\
a^{\prime \prime} & b^{\prime \prime}
\end{array}\right)
$$

for $M, M^{\prime} \in S L(2, R)$. We then find

$$
\begin{aligned}
& \text { (i) } a\left(\frac{c^{\prime} t+d^{\prime}}{a^{\prime} t+b^{\prime}}\right)+b=\frac{a^{\prime \prime} t+b^{\prime \prime}}{a^{\prime} t+b^{\prime}}, \quad c\left(\frac{c^{\prime} t+d^{\prime}}{a^{\prime} t+b^{\prime}}\right)+d=\frac{c^{\prime \prime} t+d^{\prime \prime}}{a^{\prime} t+b^{\prime}}, \\
& \text { (ii) } \frac{a}{\left(a^{\prime} t+b^{\prime}\right)\left(a^{\prime \prime} t+b^{\prime \prime}\right)}=\frac{a^{\prime \prime}}{a^{\prime \prime} t+b^{\prime \prime}}-\frac{a^{\prime}}{a^{\prime} t+b^{\prime}}, \\
& \text { (iii) } a^{\prime} t+b^{\prime}=c\left(a^{\prime \prime} t+b^{\prime \prime}\right)-a\left(c^{\prime \prime} t+d^{\prime \prime}\right) \\
& c^{\prime} t+d^{\prime}=-d\left(a^{\prime \prime} t+b^{\prime \prime}\right)+b\left(c^{\prime \prime} t+d^{\prime \prime}\right) .
\end{aligned}
$$

Also, for the proof at Eqs. (3.17), it is more convenient to rewrite Eq. (3.16) in the matrix notation of

$$
4 k \omega\left(\Lambda, \Lambda^{\prime}\right)=\left(\begin{array}{l}
\mu \\
\nu
\end{array}\right)^{T} J M\left(\begin{array}{l}
\mu^{\prime} \\
\nu^{\prime}
\end{array}\right)
$$

where $\left(\begin{array}{c}\mu \\ \nu\end{array}\right)^{T}$ is the transpose of $\left(\begin{array}{c}\mu \\ \nu\end{array}\right)$, and $J$ is given by

$$
J=\left(\begin{array}{cc}
0 & 1 \\
-1 & 0
\end{array}\right)
$$

which satisfies 


$$
M^{T} J M=M J M^{T}=J
$$

for any $M \in S L(2, R)$ and its transpose matrix $M^{T}$.

Rewriting $U$ and $W$ of section 1 as $U\left(\Lambda^{-1}\right)$ and $W\left(\Lambda^{-1}\right)$ so that

$$
U\left(\Lambda^{-1}\right) \psi(Z)=\psi^{\prime}(Z)=K(Z \mid \Lambda) \psi\left(Z^{\prime}\right),
$$

Proposition 3.2 immediately leads to:

\section{Corollary 3.3}

We have

$$
\begin{aligned}
U\left(\Lambda \circ \Lambda^{\prime}\right) & =\exp \left\{-\omega\left(\Lambda, \Lambda^{\prime}\right)\right\} U(\Lambda) U\left(\Lambda^{\prime}\right), \\
W\left(\Lambda \circ \Lambda^{\prime}\right) & =\exp \left\{-\omega\left(\Lambda, \Lambda^{\prime}\right)\right\} W(\Lambda) W\left(\Lambda^{\prime}\right) .
\end{aligned}
$$

In other words, both $U(\Lambda)$ and $W(\Lambda)$ offer projective representations of $G=S L(2, R)$ (S) $T_{2}(R)$. We note here that the cycle condition Eq. (3.17a) ensures the compatibility of Eqs. (3.23) with the associativity of products $U(\Lambda) U\left(\Lambda^{\prime}\right)$ and $W(\Lambda) W\left(\Lambda^{\prime}\right)$..

Before going into further detail, it may be worthwhile to make the following remark.

\section{$\underline{\text { Remark 3.4 }}$}

The group $G=S L(2, R)$ (S) $T_{2}(R)$ contains, in some sense, the Galilean group. Consider a set of all $\Lambda \in G$ of form

$$
\Lambda=\left\{\left(\begin{array}{ll}
1 & \lambda \\
0 & 1
\end{array}\right),\left(\begin{array}{l}
\mu \\
\nu
\end{array}\right)\right\}
$$

which causes the coordinate transformation,

$$
\begin{aligned}
& t \rightarrow t^{\prime}=t+\lambda \\
& x \rightarrow x^{\prime}=x+\sigma+v t
\end{aligned}
$$

with

$$
\begin{aligned}
\sigma & =\mu-\nu \lambda+k^{2} \beta \lambda^{2} \\
v & =2 k^{2} \beta \lambda-\nu
\end{aligned}
$$

by Eqs. (3.9). We note that the classical Newton's equation $m \ddot{x}=F$ is invariant under the Galilean transformation since the Newtonian force $F$ is constant for the 
linear potential. Therefore, in some sense, the group $G=S L(2, R)$ (s) $T_{2}$ together with Eqs. (3.9) may be said to be a quantum-mechanical generalization of the Galilean transform.

We will next give examples of Theorem 1.3 for the case of $V_{0}(\mathbf{x})=0$ and $V(\mathbf{x})=$ $\alpha+\beta x$. For simplicity, we set

$$
\begin{aligned}
& \mathcal{H}_{0}=\left\{\psi_{0}(t, x) \mid\left(\frac{\partial}{\partial t}-k \Delta\right) \psi_{0}(t, x)=0\right\} \\
& \mathcal{H}=\left\{\psi(t, x) \mid\left(\frac{\partial}{\partial t}-k \Delta+k \alpha+k \beta x\right) \psi(t, x)=0\right\} .
\end{aligned}
$$

First, we note that Eq. (1.12) for $V_{0}(\mathbf{x})=0$ implies $K(t, x) \in \mathcal{H}$. Solving conditions stated in Theorem 1.3, we then have two distinct solutions, corresponding to $C(x)=$ 0 or $\neq 0$. Rewriting these $K(t, x)$ now as $f_{j}(t, x)(j=1,2)$. We have:

\section{Proposition 3.5}

Let us set

$$
\begin{aligned}
& f_{1}(t, x)=\exp \left\{-k(\alpha+\beta x) t+\frac{1}{3} k^{3} \beta^{2} t^{3}\right\}, \\
& f_{2}(t, x)=\sqrt{\frac{1}{t}} \exp \left\{-k t\left(\alpha+\frac{\beta}{2} x\right)+\frac{1}{12} k^{3} \beta^{2} t^{3}-\frac{x^{2}}{4 k t}\right\},
\end{aligned}
$$

both of which are elements of $\mathcal{H}$. Then, for any $\psi_{0}(t, x) \in \mathcal{H}_{0}$, the functions defined by

$$
\psi_{j}(t, x)=f_{j}(t, x) \psi_{0}\left(t_{j}^{\prime}, x_{j}^{\prime}\right),(j=1,2)
$$

are elements of $\mathcal{H}$, where

$$
\begin{aligned}
& t_{1}^{\prime}=t \quad, \quad x_{1}^{\prime}=x-k^{2} \beta t^{2}, \\
& t_{2}^{\prime}=-\frac{1}{t}, \quad x_{2}^{\prime}=\frac{x}{t}-k^{2} \beta t .
\end{aligned}
$$

Conversely, suppose $\psi(t, x) \in \mathcal{H}$. Then, new functions defined by

$$
\psi_{0}^{(j)}(t, x)=\phi_{j}(t, x) \psi\left(\tilde{t}_{j}, \tilde{x}_{j}\right),(j=1,2)
$$

are elements of $\mathcal{H}_{0}$, where we have set 


$$
\begin{aligned}
& \phi_{1}(t, x)=\exp \left\{k[\alpha+\beta x] t+\frac{2}{3} k^{2} \beta^{3} t^{3}\right\} \\
& \phi_{2}(t, x)=\frac{1}{\sqrt{t}} \exp \left\{-\frac{k \alpha}{t}-\frac{2}{3} \frac{k^{2} \beta^{3}}{t^{3}}-\frac{k \beta x}{t^{2}}-\frac{x^{2}}{4 k t}\right\},
\end{aligned}
$$

with

$$
\begin{aligned}
& \tilde{t}_{1}=t \quad, \quad \tilde{x}_{1}=x+k^{2} \beta t^{2} \\
& \tilde{t}_{2}=-\frac{1}{t} \quad, \quad \tilde{x}_{2}=\frac{x}{t}+\frac{k^{2} \beta}{t^{2}} . \quad \text {. }
\end{aligned}
$$

So far, we have considered only one-dimensional problems. However, we can find some examples for multi-dimensional cases with the same symmetry group $G=$ $S L(2, R)$ (S) $T_{2}(R)$.

\section{Example 3.6}

Suppose that $\psi(t, \mathbf{x})$ with $\mathbf{x}=\left(x_{1}, x_{2}, \ldots, x_{n}\right)$ satisfy

$$
\frac{\partial}{\partial t} \psi(t, \mathbf{x})=k\left\{\Delta-\sum_{j=1}^{n}\left(\alpha+\beta x_{j}\right)-\sum_{j, k=1}^{n} \frac{a_{j k}}{\left(x_{j}-x_{k}\right)^{2}}\right\} \psi(t, \mathbf{x})
$$

where $a_{j k}$ with $a_{j j}=0$ are some constants. We also set

$$
\tilde{K}(t, \mathbf{x} \mid \Lambda)=\prod_{j=1}^{n} K\left(t, x_{j} \mid \Lambda\right)
$$

where $K(t, x \mid \Lambda)$ is given by Eqs. (3.4) and (3.5). Moreover, we consider the transformation

$$
\psi(t, \mathbf{x}) \rightarrow \psi^{\prime}(t, \mathbf{x})=\tilde{K}(t, \mathbf{x} \mid \Lambda) \psi\left(t^{\prime}, \mathbf{x}^{\prime}\right)
$$

where $t^{\prime}$ and $x_{j}^{\prime}$ are given by Eqs. (3.9) by replacing $x$ there by $x_{j}^{\prime}$ for each $j=$ $1,2, \ldots, n$. When we note

$$
x_{j}^{\prime}-x_{k}^{\prime}=\frac{1}{a t+b}\left(x_{j}-x_{k}\right)
$$

for $j, k=1,2, \ldots, n$, we can readily verify the validity of Eq. (1.9) so that $\psi^{\prime}(t, \mathbf{x})$ is another solution of Eq. (3.33). 


\section{Example 3.7}

As we will see below, the 2-dimensional nonlinear Schrödinger equation (see references 4 , and 7-9 on the subject)

$$
\frac{\partial}{\partial t} \psi\left(t, x_{1}, x_{2}\right)=k\left\{\frac{\partial^{2}}{\partial x_{1}^{2}}+\frac{\partial^{2}}{\partial x_{2}^{2}}+\lambda\left|\psi\left(t, x_{1}, x_{2}\right)\right|^{2}\right\} \psi\left(t, x_{1}, x_{2}\right)
$$

possesses also $G=S L(2, R)$ (S $T_{2}(R)$ symmetry in spite of the non-linearity of Eq. (3.36), provided that the parameter $k$ is purely imaginary. Let $\tilde{K}\left(t, x_{1}, x_{2}\right)$ be given again by Eq. (3.34) with $\alpha=\beta=0$ for $n=2$ so that

$$
\begin{aligned}
\tilde{K}\left(t, x_{1}, x_{2} \mid \Lambda\right)= & \frac{1}{a t+b} \exp \left\{-\frac{1}{2 k} \mu \nu+\frac{\nu^{2}}{2 k} \frac{c t+d}{a t+b}\right. \\
& \left.-\frac{\nu}{2 k} \frac{1}{a t+b}\left(x_{1}+x_{2}\right)-\frac{1}{4 k} \frac{a}{a t+b}\left(x_{1}^{2}+x_{2}^{2}\right)\right\} .
\end{aligned}
$$

We note then that we have

$$
\left|\tilde{K}\left(t, x_{1}, x_{2} \mid \Lambda\right)\right|^{2}=\frac{1}{(a t+b)^{2}}
$$

if $k$ is purely imaginary. Then, the new function $\psi^{\prime}\left(t, x_{1}, x_{2}\right)$ given by Eq. (3.35) for $n=2$ also satisfies

$$
\left|\psi^{\prime}\left(t, x_{1}, x_{2}\right)\right|^{2}=\frac{1}{(a t+b)^{2}}\left|\psi\left(t^{\prime}, x_{1}^{\prime}, x_{2}^{\prime}\right)\right|^{2} .
$$

As the consequence, it satisfies the analogue of Eq. (1.9), i.e.,

$$
\begin{aligned}
\left\{\frac{\partial}{\partial t}\right. & \left.-k \Delta+k \lambda\left|\psi^{\prime}\left(t, x_{1}, x_{2}\right)\right|^{2}\right\} \psi^{\prime}\left(t, x_{1}, x_{2}\right) \\
& =\frac{1}{(a t+b)^{2}} \tilde{K}\left(t, x_{1}, x_{2}\right)\left\{\frac{\partial}{\partial t^{\prime}}-k \Delta^{\prime}+k \lambda\left|\psi\left(t^{\prime}, x_{1}^{\prime}, x_{2}^{\prime}\right)\right|^{2}\right\} \psi\left(t^{\prime}, x_{1}^{\prime}, x_{2}^{\prime}\right) \\
& =0 .
\end{aligned}
$$

\section{$\underline{\text { Remark 3.8 }}$}

Let us return now to the function $f_{1}(t, x)$ given by Eq. (3.27a). One thing interesting about this function is that it is intimately related to a bound state problem. Consider the eigenvalue problem of 


$$
\left\{-\frac{d^{2}}{d x^{2}}+\beta x\right\} u(x)=E u(x)
$$

with the boundary condition $u(0)=0$ at $x=0$ for some eigenfunction $u(x) \in$ $L^{2}(0, \infty)$. To see the connection, we first note

$$
\frac{\partial}{\partial t} f_{1}(t, x)=k\left\{\frac{\partial^{2}}{\partial x^{2}}-(\alpha+\beta x)\right\} f_{1}(t, x)
$$

since $f_{1}(t, x) \in \mathcal{H}$. We next choose $k=-i$, and observe

$$
\lim _{t \rightarrow \pm \infty} f_{1}(t+i \delta, x)=0
$$

for any $\delta>0$. Therefore, if we set

$$
u(x)=\int_{-\infty}^{\infty} d t f_{1}(t+i \delta, x)
$$

and integrate Eq. (3.39) in $t, u(x)$ satisfies Eq. (3.38) with $E=-\alpha$. Moreover, letting $\delta \rightarrow+0$, we calculate

$$
u(x)=2 \int_{0}^{\infty} d t \cos \left\{(\alpha+\beta x) t+\frac{1}{3} \beta^{2} t^{3}\right\}
$$

which is the Airy's function ${ }^{10)}$ with $u(x) \in L^{2}(0, \infty)$ for $\beta>0$. Therefore, if we set $x=0$ with $\alpha=-E$, the boundary condition $u(0)=0$ leads to

$$
\int_{0}^{\infty} d t \cos \left(\frac{1}{3} \beta^{2} t^{3}-E t\right)=0
$$

The relevance of this solution to the quarquonium spectra for the $S$-wave bound states of the 3-dimensional confining linear potential can be found in ref. 11.

\section{Remark 3.9}

Another interesting property of the function $f_{1}(t, x)$ is that it is invariant under the following 2-dimensional Abelian sub-group $G_{0}$ of $G$, which consists of all elements of form:

$$
\Lambda_{0}=\left\{\left(\begin{array}{ll}
1 & \lambda \\
0 & 1
\end{array}\right),\left(\begin{array}{l}
\mu \\
0
\end{array}\right)\right\} .
$$

Then, it is straightforward to show the validity of

$$
U\left(\Lambda_{0}\right) f_{1}(t, x)=f_{1}(t, x)
$$

so that the $G$-orbit of $f_{1}(t, x)$ is the symmetric space $G / G_{0}$. Note that under $\Lambda_{0}$, the coordinate transform as a special Galilean transformation of 


$$
\begin{aligned}
t \rightarrow t^{\prime} & =t+\lambda, \\
x \rightarrow x^{\prime} & =x+\left(\mu+k^{2} \beta \lambda^{2}\right)+2 k^{2} \beta \lambda t
\end{aligned}
$$

by Eqs. (3.25).

\section{Remark 3.10}

If we set $\mu=\nu=0$, then the group $G$ reduces to $S L(2, R)$. Consider now the following time-dependent potential

$$
V(t, x)=\alpha+\beta x+\frac{\lambda}{\left(x-k^{2} \beta t^{2}\right)^{2}}
$$

for a constant $\lambda$. Then, the wave function $\psi(t, x)$ satisfying

$$
\frac{\partial}{\partial t} \psi(t, x)=k\left\{\frac{\partial^{2}}{\partial x^{2}}-V(t, x)\right\} \psi(t, x)
$$

is still invariant under the $S L(2, R)$ symmetry, since we will have

$$
x^{\prime}-k^{2} \beta t^{\prime 2}=\frac{1}{a t+b}\left(x-k^{2} \beta t^{2}\right)
$$

under Eqs. (3.9) with $\mu=\nu=0$. In section 5, we will also show that any function $\psi(t, x)$ satisfying Eq. (3.45) is an eigenstate of the Casimir invariant $I_{2}$ of the $s \ell(2)$ Lie algebra.

\section{Quadratic Potential}

The same method given in the previous sections is also applicable to the case of the quadratic potential

$$
\frac{\partial}{\partial t} \psi(t, x)=k\left\{\frac{\partial^{2}}{\partial x^{2}}-\left(\alpha+\omega^{2} x^{2}\right)\right\} \psi(t, x),
$$

for real constants $\alpha$ and $\omega^{2}$. The value of $\omega^{2}$ could also assume a negative value in what follows. However, in order to avoid the question of the reality constraint for $t^{\prime}$ and $x^{\prime}$, we will temporarily suppose that variables $t, x, t^{\prime}$, and $x^{\prime}$ as well as other parameters are allowed to assume complex values. We now perform the coordinate transformation

$$
\begin{aligned}
t \rightarrow t^{\prime} & =\phi(t), \\
x \rightarrow x^{\prime} & =\xi(t) x+f(t)
\end{aligned}
$$


as before with

$$
\begin{aligned}
\psi(t, x) & \rightarrow \psi^{\prime}(t, x)=K(t, x) \psi\left(t^{\prime}, x^{\prime}\right) \\
K(t, x) & =\exp \left\{A(t)+B(t) x+C(t) x^{2}\right\}
\end{aligned}
$$

as in section 1. Then Eqs. (1.17) and (1.18) for $n=1$ give differential equations

$$
\begin{aligned}
& \text { (i) } \xi(t) \ddot{\xi}(t)-2 \dot{\xi}(t) \dot{\xi}(t)=4 k^{2} \omega^{2} \xi^{2}(t)\left\{\xi^{4}(t)-1\right\} \\
& \text { (ii) } \xi(t) \ddot{f}(t)-2 \dot{\xi}(t) \dot{f}(t)=4 k^{2} \omega^{2} \xi^{5}(t) f(t) \\
& \text { (iii) } \dot{\phi}(t)=\xi^{2}(t)
\end{aligned}
$$

among many others.

First, the solution of Eq. (4.4a) leads to

$$
\xi^{2}(t)= \pm \frac{\exp (4 k \omega t)}{\{b+a \exp (4 k \omega t)\}\{d+c \exp (4 k \omega t)\}}
$$

for constants $a, b, c$, and $d$ satisfying $b c-a d=1$. At first glance, this appears rather peculiar, since the 2nd order differential equation, Eq. (4.4a) admits solutions containing 3 instead of 2 arbitrary parameters. However, in writing Eq. (4.5), we took advantage of the translation invariance of Eq. (4.4a) under

$$
t \rightarrow t^{\prime}=t+\text { constant }
$$

which adds one more parameter in theory. Then, the general solution of Eq. (4.4b) is found to be

$$
f(t)=\nu\left[\frac{d+c \exp (4 k \omega t)}{b+a \exp (4 k \omega t)}\right]^{\frac{1}{2}}-\mu\left[\frac{b+a \exp (4 k \omega t)}{d+c \exp (4 k \omega t)}\right]^{\frac{1}{2}},
$$

for additional constant $\mu$ and $\nu$. Therefore, the solution contains 5 parameters which we specify by

$$
\begin{aligned}
& \Lambda=\left\{M,\left(\begin{array}{l}
\mu \\
\nu
\end{array}\right)\right\} \\
& M=\left(\begin{array}{ll}
c & d \\
a & b
\end{array}\right), \text { at } M=1
\end{aligned}
$$

just as Eqs. (3.3). If we allow complex values for all these parameters, then the pre- 
sent theory remarkably gives the same symmetry group of $S L(2, C)$ (s) $T_{2}(C)$ also as we will see below. However if we restrict ourselves to real values for $t, x, t^{\prime}$, and $x^{\prime}$, then we will have the following complications. Because of the square roots operations for $\xi(t)$ in Eq. (4.5) as well as in $f(t)$ of Eq (4.6), $x^{\prime}$ given by Eq. (4.2b) will not remain real for arbitrary real values of $a, b, c, d, \mu$ and $\nu$. We will discuss the problem later.

Since all functions $\xi(t), f(t)$ etc. depend upon the parameters of $\Lambda$, we rewrite them again as $\xi(t \mid \Lambda), f(t \mid \Lambda)$ etc. However, the formulae become simpler, if we use the new variable

$$
\begin{gathered}
u=\exp (4 k \omega t) \\
u^{\prime}=\exp \left(4 k \omega t^{\prime}\right)
\end{gathered}
$$

instead of $t$ and $t^{\prime}$.

We can then rewrite Eq. (4.2) as

$$
\begin{aligned}
u^{\prime} & =\frac{c u+d}{a u+b} \quad, \quad b c-a d=1 \\
x^{\prime} & =\xi(t \mid \Lambda) x+f(t \mid \Lambda)
\end{aligned}
$$

where

$$
\begin{aligned}
& \xi(t \mid \Lambda)=\left[\frac{u}{(a u+b)(c u+d)}\right]^{\frac{1}{2}}, \\
& f(t \mid \Lambda)=\nu\left[\frac{c u+d}{a u+b}\right]^{\frac{1}{2}}-\mu\left[\frac{a u+b}{c u+d}\right]^{\frac{1}{2}} .
\end{aligned}
$$

Calculating now explicit forms of $A(t \mid \Lambda), B(t \mid \Lambda)$, and $C(t \mid \Lambda)$ as in the previous section, we find:

\section{Proposition 4.1}

For any $\psi(t, x)$ satisfying Eq. (4.1), the new function given by

$$
\psi^{\prime}(t, x)=K(t, x \mid \Lambda) \psi\left(t^{\prime}, x^{\prime}\right)\left(=U\left(\Lambda^{-1}\right) \psi(t, x)\right)
$$

is also a solution of the same differential equation, Eq. (4.1). Here, we have set 


$$
\begin{aligned}
K(t, x \mid \Lambda)= & \exp \left\{A(t \mid \Lambda)+B(t \mid \Lambda) x+C(t \mid \Lambda) x^{2}\right\}, \\
A(t \mid \Lambda)= & \frac{1}{4} \log \frac{u}{(a u+b)(c u+d)}+\frac{\alpha}{4 \omega}\left\{\log \left(\frac{c u+d}{a u+b}\right)-\log u\right\} \\
& +\frac{\omega}{2}\left\{\nu^{2} \frac{c u+d}{a u+b}-\mu^{2} \frac{a u+b}{c u+d}\right\}, \\
B(t \mid \Lambda)= & \omega\left\{\frac{\nu \sqrt{u}}{a u+b}+\frac{\mu \sqrt{u}}{c u+d}\right\}, \\
C(t \mid \Lambda)= & \frac{\omega}{2}\left\{-1+\frac{b}{a u+b}+\frac{d}{c u+d}\right\} .
\end{aligned}
$$

We introduce the product $\Lambda \circ \Lambda^{\prime}$ for two $\Lambda$ and $\Lambda^{\prime}$ again by Eq. (3.11), which defines the group $G=S L(2, C)$ (S) $T_{2}(C)$ for complex $\Lambda$ and $\Lambda^{\prime}$. We also assume the action of $\Lambda$ to the coordinate $Z=\{t, x\}$ to be given by Eqs. (3.14) so that

$$
\Lambda Z=\Lambda\{t, x\}=\left\{t^{\prime}, x^{\prime}\right\}
$$

We then discover after some calculations that the exact analogue of Proposition 3.2 also holds valid for the present case except for the fact that $\omega\left(\Lambda, \Lambda^{\prime}\right)$ there is now replaced by

$$
\omega\left(\Lambda, \Lambda^{\prime}\right) \rightarrow \tilde{\omega}\left(\Lambda, \Lambda^{\prime}\right)=\omega\left\{(\mu a-\nu c) \mu^{\prime}+(\mu b-\nu a) \nu^{\prime}\right\} .
$$

For the proof of these facts, the identities Eqs. (3.19) (with $t \rightarrow u$ ) as well as

$$
\begin{aligned}
& \frac{b}{a^{\prime \prime} u+b^{\prime \prime}} \frac{a^{\prime} u+b^{\prime}}{c^{\prime} u+d^{\prime}}=\frac{1}{c^{\prime} u+d^{\prime}}-\frac{a}{a^{\prime \prime} u+b^{\prime \prime}} \\
& \frac{d}{c^{\prime \prime} u+d^{\prime \prime}} \frac{a^{\prime} u+b^{\prime}}{c^{\prime} u+d^{\prime}}=\frac{1}{c^{\prime} u+d^{\prime}}-\frac{c}{c^{\prime \prime} u+d^{\prime \prime}}
\end{aligned}
$$

are useful, although we will not go into detail. However, we do not understand the reason why both cases of linear and quadratic potentials give at least formally the identical final results.

\section{$\underline{\text { Remark } 4.2}$}

In contrast to the case of linear potential, the special transformation Eq. (3.24) of $G=S L(2, R)$ (s) $T_{2}(R)$ with Eq. (4.9) does not give the Galilean formula Eqs. (3.25) for the present problem. This is, of course, expected since the classical Newton's formula $m \ddot{x}=F=\lambda x$ (sor some constant $\lambda$ ) is no longer invariant under the Galilean transformation. 
So far we have ignored the question of the reality for variables $t, x, t^{\prime}$, and $x^{\prime}$. Let us discuss the problem in some details below. Since both constants $k$ and $\omega$ are assumed to be either real or purely imaginary, so will be the product $k \omega$. Suppose first that $k \omega$ is real. Then, $u=\exp (4 k \omega t)$ is real and positive for real $t$. The condition that both $x^{\prime}$ and $t^{\prime}$ are real requires that $a u+b$ and $c u+d$ be real with $(a u+b)(c u+d)>0$ for any $u>0$. This can be possible in general only in a neighborhood of the unit element $E=\left(\begin{array}{ll}1 & 0 \\ 0 & 1\end{array}\right)$ of the $S L(2, R)$ matrix $M$. Moreover, the allowed values for $M$ depend upon the time $t$. In other words, the symmetry group of the theory is not in general global $S L(2, R)$ group, but is the so-called local group (or group germ). Alternatively we may better consider a sub-set of $S L(2, R)$ such that all $a, b, c, d$ are non-negative. Then, the reality condition for $t^{\prime}$ and $u^{\prime}$ are readily maintained. However, the inverse matrix $M^{-1}$ does not satisfy the requirement, then. In this case, the symmetry is not a group but a global semi-group consisting of all non-negative matrices in $S L(2, R)$, when $k \omega$ is real. Note that $\mu$ and $\nu$ are chosen to be real in the present case in order to make $x^{\prime}$ to be real in Eq. (4.9b).

On the other side, suppose now that $k \omega$ is purely imaginary. Then, we have $|u|=\left|u^{\prime}\right|=1$. In that case, instead of the parametrization Eq. (4.7b) for $M$, we may use the conformal mapping in the complex $u$-plane by

$$
u \rightarrow u^{\prime}=e^{2 i \theta} \frac{u-\lambda}{1-\lambda^{*} u}
$$

for real $\theta$ and any complex $\lambda$ with $|\lambda| \neq 1$. The condition $\left|u^{\prime}\right|=1$ whenever we have $|u|=1$ is automatically guaranteed by Eq. (4.16) for arbitrary complex number $\lambda$. In terms of $\theta$ and $\lambda$, we can express $a, b, c, d$ as

$$
\begin{aligned}
& a=-\frac{\lambda^{*}}{\sqrt{1-|\lambda|^{2}}} e^{i \theta} \quad, \quad b=\frac{1}{\sqrt{1-|\lambda|^{2}}} e^{-i \theta}, \\
& c=\frac{1}{\sqrt{1-|\lambda|^{2}}} e^{i \theta}=b^{*} \quad, \quad d=-\frac{\lambda}{\sqrt{1-|\lambda|^{2}}} e^{-i \theta}=a^{*}
\end{aligned}
$$

for $|\lambda|<1$. This especially gives a identity

$$
c u+d=(a u+b)^{*} u
$$

for $|u|=1$. Then, Eq. (4.10a) gives the desired result of the reality constraint of

$$
\xi(t \mid \Lambda)=\frac{1}{|a u+b|}>0
$$

If we next rewrite Eq. (4.10b) as

$$
f(t \mid \Lambda)=\nu\left[\frac{(a u+b)^{*} u}{a u+b}\right]^{\frac{1}{2}}-\mu\left[\frac{(a u+b) u^{*}}{(a u+b)^{*}}\right]^{\frac{1}{2}}
$$


for $|u|=1$, then the reality of $f(t \mid \Lambda)$ can also be maintained as long as we have

$$
\mu^{*}=-\nu
$$

We can verify that both Eq. (4.20) and $M=\left(\begin{array}{cc}b^{*}, & a^{*} \\ a, & b\end{array}\right)$ given by Eq.

remains invariant under the composition law Eq. (3.11). In conclusion, if $k \omega$ is purely imaginary, the symmetry group of the problem is a particular global sub-group of $G=S L(2, C)$ S $T_{2}(C)$, where we use the parametrization of $S L(2, C)$ and $T_{2}(C)$ as in Eqs. (4.17) and (4.20). Especially, it contains a group of conformal mappings of transforming the unit circle onto itself in the complex $u$-plane.

Last, we would like to present another example for Theorem 1.3 for the present problem with $V_{0}(\mathbf{x})=0$. Let us set

$$
\begin{aligned}
& \mathcal{H}_{0}=\left\{\psi_{0}(t, x) \mid\left(\frac{\partial}{\partial t}-k \frac{\partial^{2}}{\partial x^{2}}\right) \psi_{0}(t, x)=0\right\} \\
& \mathcal{H}_{1}=\left\{\psi(t, x) \mid\left(\frac{\partial}{\partial t}-k \frac{\partial^{2}}{\partial x^{2}}-k\left(\alpha+\omega^{2} x^{2}\right)\right) \psi(t, x)=0\right\}
\end{aligned}
$$

Solving conditions given in Theorem 1.3, we then find:

\section{Proposition 4.2}

For any $\psi_{0}(t, x) \in \mathcal{H}_{0}$, the function given by

$$
\psi(t, x)=K_{0}(t, x) \psi_{0}\left(t^{\prime}, x^{\prime}\right)
$$

is an element of $\mathcal{H}$, where

$$
\begin{aligned}
t^{\prime} & =-\frac{\sigma^{2}}{4 k \omega} \frac{1}{u+\lambda} \\
x^{\prime} & =\sigma \frac{\sqrt{u}}{u+\lambda} x-\frac{\sigma \tau}{2 \omega} \frac{1}{u+\lambda},
\end{aligned}
$$

with

$$
\begin{aligned}
K_{0}(t, x) & =\frac{u^{\frac{1}{4}}}{(\lambda+u)^{\frac{1}{2}}} \exp \left\{A_{0}(t)+B_{0}(t) x+C_{0}(t) x^{2}\right\} \\
A_{0}(t) & =-\frac{\tau^{2}}{4 \omega} \frac{1}{u+\lambda}-k \alpha t \quad
\end{aligned}
$$




$$
\begin{aligned}
& B_{0}(t)=\tau \frac{\sqrt{u}}{u+\lambda}, \\
& C_{0}(t)=\frac{\omega(\lambda-u)}{2(u+\lambda)}
\end{aligned}
$$

Here, $\sigma, \lambda, \tau$ are arbitrary constants and

$$
u=\exp \{4 k \omega t\}
$$

\section{$\underline{\text { Remark } 4.3}$}

Unfortunately, the variables $t^{\prime}$ and $x^{\prime}$ given by Eqs. (4.23) can be real only for the case of $k \omega$ being real. For that case, we can construct solutions of Eq. (4.21b) from that of $\psi(t, x)$ satisfying Eq. (3.26b) by combining Eqs. (3.30) and (4.22).

\section{$5 \quad$ Lie Algebras and Local Symmetry}

In the preceding sections, we found that the time-dependent Schrödinger equation for some potentials has global groups as symmetry of the theory. However, much larger local symmetry could emerge, if we consider its Lie algebraic structure as follows.

Let us first set for simplicity

$$
K \equiv \frac{\partial}{\partial t}-k\left\{\frac{\partial^{2}}{\partial x^{2}}-V(x)\right\}
$$

and

$$
\mathcal{H}=\{\psi(t, x) \mid K \psi(t, x)=0\}
$$

Consider now, as an example, the symmetry group $G=S L(2, R)$ (s) $T_{2}(R)$ of section 3 for the linear potential. We know that for any $\psi(t, x) \in \mathcal{H}$, we have $U(\Lambda) \psi(t, x) \in \mathcal{H}$ for any $\Lambda \in G$. Since $G$ is a Lie group, we can associate a Lie algebra $L$ by considering infinitesimal $\Lambda$ 's. It is then evident that we have

$$
g \psi(t, x) \in \mathcal{H} \quad, \quad g \in L \quad .
$$

Let $\tilde{U}(L)$ be the universal enveloping algebra of $L$. Also, we will then have

$$
\tilde{U}(L) \psi(t, x) \in \mathcal{H}
$$


whenever $\psi(t, x)$ is a sufficiently smooth function of $t$ and $x$. Since $K$ is invariant under the time translation $t \rightarrow t^{\prime}=t+\lambda$ for any constant $\lambda$, the Lie algebra $L$ always contain a special element

$$
D \equiv \frac{\partial}{\partial t} \in L
$$

so that this implies the validity of

$$
D^{n} \psi(t, x) \in \mathcal{H}
$$

for any positive integer $n$. This can be, of course, more directly verified from $\left[D^{n}, K\right]=$ 0 . For the case of the linear potential of section 3, the Lie algebra $L$ consists now of 6 elements (instead of 5 with the additional unit element 1);

$$
L=\left\{L_{ \pm}, L_{3}, T_{1}, T_{2}, 1\right\}
$$

which forms the Abelian-extended Lie algebra of

$$
L=s \ell(2) \oplus t(2) \oplus u(1)
$$

where $u(1)$ is the extra one-dimensional Abelian algebra in conformity with the projective representation nature of $U(\Lambda)$ in Eqs. (3.23). Their explicit forms are easily calculated from Eqs. (3.4), (3.5), (3.7) and (3.9) to be given by

$$
\begin{aligned}
-L_{3}= & t \frac{\partial}{\partial t}+\frac{1}{2}\left(x+3 k^{2} \beta t^{2}\right) \frac{\partial}{\partial x}+k\left(\alpha+\frac{3}{2} \beta x\right) t+\frac{1}{2} k^{3} \beta^{2} t^{3}+\frac{1}{4} \\
L_{+}= & \frac{\partial}{\partial t}+2 k^{2} \beta t \frac{\partial}{\partial x}+k(\alpha+\beta x)+k^{3} \beta^{2} t^{2}, \\
L_{-}= & t^{2} \frac{\partial}{\partial t}+\left(t x+k^{2} \beta t^{3}\right) \frac{\partial}{\partial x}+\frac{1}{2} t+\alpha k t^{2} \\
& +\frac{1}{4} k^{3} \beta^{2} t^{4}+\frac{3}{2} k \beta t^{2} x+\frac{1}{4 k} x^{2}
\end{aligned}
$$

and

$$
\begin{aligned}
& T_{1}=\frac{\partial}{\partial x}+k \beta t \\
& T_{2}=t \frac{\partial}{\partial x}+\frac{1}{2 k} x+\frac{k \beta}{2} t^{2} .
\end{aligned}
$$

They satisfy commutation relations: 


$$
\begin{aligned}
{\left[L_{3}, L_{ \pm}\right] } & = \pm L_{ \pm} \quad, \quad\left[L_{+}, L_{-}\right]=-2 L_{3} \\
{\left[L_{3}, T_{1}\right] } & =\frac{1}{2} T_{1} \quad, \quad\left[L_{3}, T_{2}\right]=-\frac{1}{2} T_{2} \\
{\left[L_{+}, T_{1}\right] } & =\left[L_{-}, T_{2}\right]=0 \quad \\
{\left[L_{+}, T_{2}\right] } & =T_{1} \quad, \quad\left[L_{-}, T_{1}\right]=-T_{2} \\
{\left[T_{1}, T_{2}\right] } & =\frac{1}{2 k}
\end{aligned}
$$

Note that $\left[T_{1}, T_{2}\right]=\frac{1}{2 k} \neq 0$, reflecting the projective representation of Eq. (3.23). We note that $T_{1}$ and $T_{2}$ play the role of creation and annihilation operators.

Since $W(\Lambda)$ is also a representation of $G$, we can perform the same analysis to find that the corresponding Lie algebra $\tilde{L}$ consisting of

$$
\tilde{L}=\left\{\tilde{L}_{ \pm}, \tilde{L}_{3}, \tilde{T}_{1}, \tilde{T}_{2}, 1\right\}
$$

has the form

$$
\begin{aligned}
& \tilde{L}_{3}=L_{3}-1 \quad, \quad \tilde{L}_{+}=L_{+} \quad, \quad \tilde{L}_{-}=L_{-}+2 t \\
& \tilde{T}_{1}=T_{1} \quad, \quad \tilde{T}_{2}=T_{2}
\end{aligned}
$$

with the same commutation relation, Eqs. (5.11). When we write

$$
K_{1}=\frac{\partial}{\partial t}-k\left\{\frac{\partial^{2}}{\partial x^{2}}-\alpha-\beta x\right\},
$$

then Eq. (1.11) becomes

$$
\tilde{L} K_{1}=K_{1} L
$$

This especially implies $\left[L_{+}, K_{1}\right]=\left[T_{1}, K_{1}\right]=\left[T_{2}, K_{1}\right]=0$ and $\left[L_{3}, K_{1}\right]=K_{1}$. Moreover, we can easily find that $K_{1}$ is rewritten as a element of $\tilde{U}(L)$ as

$$
K_{1}=L_{+}-k T_{1}^{2}
$$

while the time derivative $D=\frac{\partial}{\partial t}$ is expressed as

$$
D=L_{+}-2 k^{2} \beta T_{2}-k \alpha
$$

Before going into further details, we note first that the second order Casimir invariant of the $s \ell(2)$ sub-Lie algebra of $L$ is given by

$$
I_{2}=L_{+} L_{-}-L_{3}^{2}+L_{3} \quad
$$


In contrast, the larger Lie algebra $L$ possesses not the second but third order Casimir invariant

$$
I_{3}=-I_{2}+k\left\{L_{3}\left(T_{1} T_{2}+T_{2} T_{1}\right)+L_{+} T_{2} T_{2}+L_{-} T_{1} T_{1}\right\} \quad .
$$

For our particular form of generators given by Eqs. (5.9) and Eq. (5.10), we find that $I_{3}$ is purely a constant

$$
I_{3}=\frac{3}{16}
$$

while $I_{2}$ is rewritten as

$$
I_{2}=\frac{3}{16}+\frac{1}{4 k}\left(x-k^{2} \beta t^{2}\right)^{2} K_{1}
$$

Especially for any function $\psi=\psi(t, x)$ satisfying $K_{1} \psi=0$, we have $I_{2} \psi=\frac{3}{16} \psi$. In this connection, two special functions $f_{1}(t, x)$ and $f_{2}(t, x)$ given in Eqs. (3.27) have the following interesting property. They satisfy

$$
\begin{array}{ll}
L_{+} f_{1}(t, x)=T_{1} f_{1}(t, x)=0 \quad, \quad L_{3} f_{1}(t, x)=-\frac{1}{4} f_{1}(t, x) \\
L_{-} f_{2}(t, x)=T_{2} f_{2}(t, x)=0 \quad, \quad L_{3} f_{2}(t, x)=\frac{1}{4} f_{2}(t, x),
\end{array}
$$

Therefore, the function $f_{1}(t, x)$ corresponds to the highest weight state of simultaneous representations of both $s \ell(2)$ and $L$, while $f_{2}(t, x)$ plays the role of the lowest weight state of another representation. They are infinite dimensional and irreducible but not unitary. Moreover, they satisfy $K_{1} f_{j}(t, x)=0$ for $j=1,2$ so that we have

$$
I_{2} f_{j}(t, x)=I_{3} f_{j}(t, x)=\frac{3}{16} f_{j}(t, x) \quad, \quad(j=1,2)
$$

Also, in view of Eq. (5.20), the wave function $\psi(t, x)$ satisfying Eqs. (3.44) and (3.45) is the eigenstate of $I_{2}$ with $I_{2} \psi(t, x)=\left\{\frac{3}{16}-\frac{\lambda}{4}\right\} \psi(t, x)$.

The same analysis is readily applicable for the quadratic potential of section 4 . In this case, the Lie algebras $L$ and $\tilde{L}$ are specified by

$$
\begin{aligned}
-L_{3} & =u \frac{\partial}{\partial u}+\frac{\alpha}{4 \omega}, \\
L_{+} & =\frac{\partial}{\partial u}-\frac{1}{2 u} x \frac{\partial}{\partial x}+\left(\frac{\alpha}{4 \omega}-\frac{1}{4}\right) \frac{1}{u}+\frac{\omega}{2} \frac{x^{2}}{u}, \\
L_{-} & =u^{2} \frac{\partial}{\partial u}+\frac{1}{2} u x \frac{\partial}{\partial x}+\left(\frac{\alpha}{4 \omega}+\frac{1}{4}\right) u+\frac{\omega}{2} u x^{2}
\end{aligned}
$$




$$
\begin{aligned}
& T_{1}=\frac{1}{\sqrt{u}} \frac{\partial}{\partial x}-\frac{\omega}{\sqrt{u}} x, \\
& T_{2}=\sqrt{u} \frac{\partial}{\partial x}+\omega \sqrt{u} x,
\end{aligned}
$$

with $u=\exp (4 k \omega t)$, and

$$
\begin{array}{ll}
\tilde{L}_{3}=L_{3} \quad, \quad \tilde{L}_{+}=L_{+}-\frac{1}{u} \quad, \quad \tilde{L}_{-}=L_{-}+u, \\
\tilde{T}_{1}=T_{1} \quad, \quad \tilde{T}_{2}=T_{2} .
\end{array}
$$

They satisfy the same commutation relations. Eqs. (5.11a-5.11d) while Eqs. (5.11e) is now replaced by

$$
\left[T_{1}, T_{2}\right]=2 \omega
$$

Writing

$$
K_{2}=\frac{\partial}{\partial t}-k\left\{\frac{\partial^{2}}{\partial x^{2}}-\alpha-\omega^{2} x^{2}\right\} \quad,
$$

the analogues of Eqs. (5.15), and (5.16), are now given by

$$
\begin{aligned}
\tilde{L} K_{2} & =K_{2} L \\
K_{2} & =-4 k \omega L_{3}-\frac{k}{2}\left(T_{1} T_{2}+T_{2} T_{1}\right) \\
D & =\frac{\partial}{\partial t}=-4 k \omega L_{3}-k \alpha .
\end{aligned}
$$

Especially, Eqs. (5.24) and (5.27a) lead to

$$
\left[L_{3}, K_{2}\right]=\left[T_{1}, K_{2}\right]=\left[T_{2}, K_{2}\right]=0 .
$$

For this case, the 2nd order Casimir invariant $I_{2}$ is still given by Eq. (5.17), while Eq. (5.18) for $I_{3}$ must now be replaced by

$$
I_{3}=-I_{2}+\frac{1}{4 \omega}\left\{L_{3}\left(T_{1} T_{2}+T_{2} T_{1}\right)+L_{-} T_{1} T_{1}+L_{+} T_{2} T_{2}\right\} .
$$

We still have the validity of $I_{3}=\frac{3}{16}$ but Eq. (5.20) is now replaced by

$$
I_{2}=\frac{3}{16}+\frac{1}{4 k} x^{2} K_{2}
$$

Especially, if $\psi(t, x)$ now satisfies 


$$
\left\{\frac{\partial}{\partial t}-k\left(\frac{\partial^{2}}{\partial x^{2}}-\alpha-\omega^{2} x^{2}-\frac{\lambda}{x^{2}}\right)\right\} \psi(t, x)=0
$$

for some constant $\lambda$, then Eq. (5.29) now implies

$$
I_{2} \psi(t, x)=\left(\frac{3}{16}-\frac{\lambda}{4}\right) \psi(t, x) \quad .
$$

There exist relations analogous to Eqs. (5.21). Setting

$$
\begin{aligned}
& g_{1}(t, x)=\exp \left\{k(\omega-\alpha) t+\frac{\omega}{2} x^{2}\right\}, \\
& g_{2}(t, x)=\exp \left\{-k(\omega+\alpha) t-\frac{\omega}{2} x^{2}\right\},
\end{aligned}
$$

it is easy to verify

$$
\begin{aligned}
& K_{2} g_{1}(t, x)=L_{+} g_{1}(t, x)=T_{1} g_{1}(t, x)=0 \quad, \quad L_{3} g_{1}(t, x)=-\frac{1}{4} g_{1}(t, x) \\
& K_{2} g_{2}(t, x)=L_{-} g_{2}(t, x)=T_{2} g_{2}(t, x)=0 \quad, \quad L_{3} g_{2}(t, x)=\frac{1}{4} g_{2}(t, x)
\end{aligned}
$$

Note that $g_{2}(t, x)$ given by Eq. (5.30b) corresponds to the ground state wave function of the familiar harmonic potential. It is again the lowest weight state of representations of both $s \ell(2)$ and $L$. Another interesting function is obtained by setting $\tau=\lambda=0$, and $\sigma=1$ with appropriate choice for $\psi_{0}(t, x)$ in Proposition 4.3. In this way, the function

$$
g_{3}(t, x)=\exp \left\{-k(\alpha+\omega) t-\frac{\omega}{2} x^{2}-\gamma \frac{x}{\sqrt{u}}-\frac{\gamma^{2}}{4 \omega} \frac{1}{u}\right\}
$$

for an arbitrary constant $\gamma$ turns out to be a simultaneous eigenfunction of $K_{2}, T_{2}$ and $L_{-}$as in

$$
\begin{aligned}
& \text { (i) } \quad K_{2} g_{3}(t, x)=0 \\
& \text { (ii) } \quad T_{2} g_{3}(t, x)=\gamma g_{3}(t, x) \\
& \text { (iii) } \quad L_{-} g_{3}(t, x)=\frac{\gamma^{2}}{4 \omega} g_{3}(t, x)
\end{aligned}
$$

Especially, Eq. (5.33b) implies that $g_{3}(t, x)$ plays a role of coherent state as in the quantum optics, ${ }^{12)}$ since $T_{2}$ may be regarded as the analogue of the annihilation operator. 


\section{$\underline{\text { Acknowledgement }}$}

This paper is supported in part by U.S. Department of Energy Contract no. DE-FG02-91ER40685. 


\section{$\underline{\text { References }}$}

1. J. Wu and Y. Alhassid, The potential group approach and hypergeometric differential equations, J. Math. Phys. 31, 557-562 (1990).

2. M. Sezgin, A.Y. Verdiyev, and Y.A. Verdiyev, Generalized Pöschl-Teller, Toda, Morse potential and $S L(2, R)$ group, J. Math. Phys. 39, 1910-1918 (1998).

3. N.M. Nieto and D.R. Truax, Time-dependent Schrödinger equations having isomorphic symmetry algebras, II. Symmetry algebra, coherent and squeezed state, J. Math. Phys. 41, 2753-2767 (2000).

4. H.D. Doebner and G. Goldin, Some remarks on non-linear quantum mechanics, a talk presented at the 24th International Colloquium on Group Theoretical Methods in Physics, held at Paris, July 15-20 (2002).

5. M. Eastwood, Symmetry and differential invariants, a talk presented at the 6th International Conference on Clifford Algebras and Their Applications held at Tennessee Technological University, Cookeville, TN, May 18-25 (2002).

6. A. Erdelyi, W. Magnus, F. Oberhettinger, and F.G. Tricomi, Higher Transcendental Functions, Bateman Manuscript Project Vol. 3, McGraw Hill (NY) 1955.

7. D. Faddev and L.A. Takhtajan, Hamiltonian methods in the theory of solitions, (Springer, Berlin 1987).

8. A. Das, Integrable Models, (World Scientific, Singapore 1989).

9. M.A. Ablowitz and P.A. Clarkson, Solitons, non-linear evolution equations and inverse scattering, (Cambridge, New York, 1991).

10. M. Abramowitz and I.A. Stegun, Handbook of Mathematical Functions, Dober Pub., NY (1970), see pp.446-447.

11. A. Das and A.C. Melissinos, Quantum Mechanics, A Modern Introduction, Gordon and Breach, NY (1986), pp.339-352.

12. L. Mandel and E. Wolf, Optical Coherence and Quantum Optics, Cambridge Univ. Press, NY (1995), pp.538-540. 